மார்துளைத்த புற்றுநோயினால் மனமுடைந்த பெண்களுக்கான உள சிகிச்சையும், தியான பயிற்சிகளும்

ச. அகிலா அ,, பொ. பிரியதர்ஷினி அ, மா. சாரு நிவேதிதா அ, இரா. திலகவதி சண்முகசுந்தரம் ஆ

அ உடற்கல்வித் துறை, பாரதியார் பல்கலைக்கழகம், கோயம்புத்தூர்-641046, தமிழ்நாடு, இந்தியா.

ஆ தமிழ் ஆசிரியை (ஓய்வு) மாநகராட்சி பெண்கள் மேல் நிலைப் பள்ளி, கோயம்புத்தூர்-641002, தமிழ்நாடு, இந்தியா.

\title{
Meditation to Mediate Emotional Swings of Breast Cancer Survivors
}

\author{
S. Akila a, ${ }^{*}$ P. Priyadharsni a, M. Charu Nivedita a, Thilagavathi Shanmuga \\ Sundaram ${ }^{b}$ \\ a Department of Physical Education, Bharathiar University, Coimbatore- 641046, Tamil Nadu, India. \\ b Tamil Teacher (Retired), Corporation Girls High School, Coimbatore- 641002, Tamil Nadu, India.
}

* Corresponding Author: akila.sathish@yahoo.com

Received : 19-08-2020

Accepted : 23-12-2020

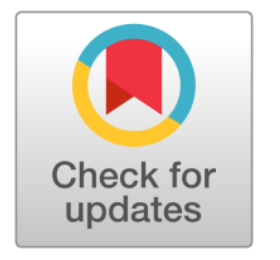

\section{ABSTRACT}

Cancer has gradually occupied a bigger space in mortality count. It's reported that every four minutes a person is affected by cancer. Women had taken over men; mainly 1 in 30 women are affected by breast cancer. Breast cancer has cancer. Breast cancer has a great impact on emotional \& social perspectives. More than an organ it's a symbol of beauty and motherhood. In Ancient Tamil literatures, mainly sanga literatures at many instances poets have glorified the beauty of women, donating breast, hair, waist line \& motherhood. Though difficult we still can survive with one kidney, one eye, leg and hand but in breast cancer it is tragedy. The pain of hair due to chemotherapy, losing a breast and beauty seems to be higher than pain caused by the disease. They undergo a lot of mood swings and emotional imbalances, to build in resilience and to enhance their livelihood meditation. Yoga helps than to beat over fatigue. It also helps manage the side effects of cancer treatment and helps manage the emotional add-ons such us mental fatigue, depression. Yoga is mainly recommended for the psycho-social wellbeing along with family counselling. The yogic package mainly had pranayama practice, meditation and few asanas. Regular practice if continued by breast cancer survivor even after the cancer cure will have lifelong benefits. The purpose of writing this article is to create awareness among women of my own motherland-Tamil Nadu to stay conscious and undergo regular screening as women are the true lights of our nation.

Keywords: Breast Cancer, Women, Yoga, 


\section{ஆசிரியர் குறிப்பு}

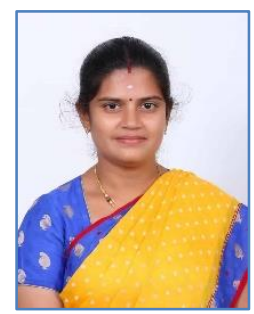

முனைவர் அகிலா ச அவர்கள் தற்போது பாரதியார் பல்கலைக்கழகத்தில் உடற்கல்வி துறையில் உதவிப் பேராசிரியராக பணியாற்றிக் கொண்டிருக்கிறார். தனது முதுகலைப் பட்டப்படிப்பை பாரதியார் பல்கலைக் கழகத்திலும் முனைவர் பட்ட ஆய்வை ராமகிருஷ்ண மிஷன் விவேகானந்தா பல்கலைக் கழகத்திலும் நிறைவு செய்துள்ளார். சிறந்த முன்னாள் தடகள வீராங்கனையான இவர் பந்தய நடை ஓட்டப் போட்டிகளில் மாநில மற்றும் தேசிய அளவில் பல பதக்கங்களை குவித்து தேசிய சாதனைகளையும் நிகழ்த்தியுள்ளார். தமிழ் நாடு அறிவியல் தொழில் நுட்ப மாநில மன்றத்தால் வழங்கப்படும் இளம் விஞ்ஞானி விருதினை 2019 மற்றும் 2020 ஆகிய இரண்டு ஆண்டுகளாக பெற்றுள்ளார். உடற்கல்வி துறையில் தமிழகத்தில் இவ்விருதினை இரண்டு முறை பெற்ற ஆய்வாளர் இவர் மட்டுமே என்பது குறிப்பிடத்தக்கது. உள்நாட்டிலும் வெளி நாட்டிலும் நடை பெற்ற பல ஆய்வுக் கருத்தரங்குகளில் பங்கு பெற்று தனது ஆய்வை சமர்ப்பித்துள்ள இவர் சிறப்பு பேச்சாளராகவும் பல சபைகளில் அங்கம் வகித்துள்ளார். இவர் பல சர்வதேச ஆய்விதழ்களில் 12-ம் மேற்பட்ட ஆய்வுக் கட்டுரைகளை வெளியிட்டுள்ளார். மேலும் பல சர்வதேச ஆய்விதழ்களின் ஆசிரியர் குழுவிலும் உள்ளார். இவர் 15 மேற்பட்ட புத்தகங்களையும் வெளியிட்டுள்ளார். தமிழின் மீது தீரக் காதல் கொண்ட இவர் தமிழில் சில விளையாட்டு தொடர்பான மற்றும் கவிதைப் புத்தகங்களையும் எழுதியுள்ளார். இது தவிர பல தன்னார்வ அமைப்புகளுடன் இ இணைந்து சமூக சேவைகளும் செய்து வருகிறார் என்பது குறிப்பிடத்தக்கது.

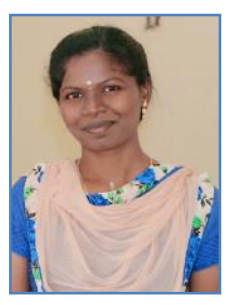

செல்வி. பொ.பிரியதர்ஷினி அவர்கள் கோவை பாரதியார் பல்கலைக்கழகத்தில் உடற்கல்வி துறையில் ஆய்வியல் நிறைஞராக பயின்று வருகிறார். கோவை அவிநாசிலிங்கம் பல்கலைக்கழகத்தில் உடற்கல்வி துறையில் இளங்கலை மற்றும் கோவை பாரதியார் பல்கலைக்கழகத்தில் உடற்கல்வி துறையில் முதுகலை பட்டத்தையும் பெற்றார். இவர் பல்கலைக் கழகத்திலேயே முதல் மதிப்பெண் பெற்று தேர்ச்சி அடைந்தார். இவர் தேசிய வாள்சண்டை விளையாட்டு வீராங்கனை ஆவார்.

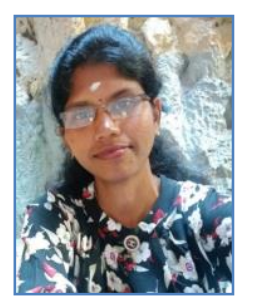

செல்வி.மா.சாருநிவேதிதா அவர்கள் கோவை பாரதியார் பல்கலைக்கழகத்தில் உடற்கல்வி துறையில் ஆராய்சி அறிஞராக பயின்று வருகிறார். கோவை பி.எஸ்.ஜி.ஆர் கிருஷ்ணமாள் கல்லூரியில் ஆங்கில துறையில் இளங்கலை மற்றும் கோவை பாரதியார் பல்கலைக்கழகத்தில் உடற்கல்வி துறையில் முதுகலை பட்டத்தையும் பெற்றார். இவர் தேசிய கூடைப்பந்து விளையாட்டு வீராங்கனை ஆவார்.

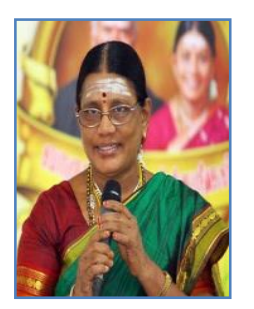

கயிலைமணி, பைந்தமிழ்ச்செல்வி முனைவர். இரா. திலகவதி சண்முகசுந்தரம் அவர்கள் 'தமிழகப் பக்தி இலக்கிய வளர்ச்சியில் மகளிர் பங்கு' என்ற பொருளில் ஆய்வு செய்து முனைவர் பட்டம் பெற்றவர். 2005 ஆம் ஆண்டின் மாநில அரசின் டாக்டர் ராதாகிருஷ்ணன் நல்லாசிரியர் விருதும் 2003 ஆம் ஆண்டு லண்டனில் சிங்கதமிழரசி என்னும் பட்டமும் பெற்றுள்ளார். தமிழாசிரியராகவும், தலைமையாசிரியராகவும் சிறப்பாகப் பணியாற்றியவர். தமிழ் மீது ஆர்வம் கொண்ட இவர் இலங்கை, லண்டன், பிரான்ஸ், சுவிட்சர்லாந்து, ஜெர்மனி, மொரிசியஸ் ஆகிய நாடுகளில் தமிழ் கருத்தரங்குகளில் கலந்துகொண்டு சொற்பொழிவாற்றியுள்ளார். பணி ஓய்விற்கு பிறகும் தமிழ் வளர்ச்சிக்காகவும் பெண்களின் மேம்பாத்திற்காகவும் பல தொண்டுகள் ஆற்றி வருகிறார். 


\section{முன்னுரை}

நான்கு நிமிடத்திற்கு ஒருவர் என மார்பகப் புற்றுநோய் மனித குலத்தையே உலுக்கிக் கொண்டிருக்கிறது. அதிலும் ஆண்களை விட பெண்களே அதிகம் தாக்கத்திற்குள்ளாகின்றனர். தோராயமாக 30-ல் ஒரு பெண் மார்பகப் புற்றுநோயினால் பாதிக்க படுவதாக மருத்துவ ஆராய்ச்சிக்கான இந்திய சபை (ICMR-2020) வெளியுட்டுள்ள கணக்கெடுப்பில் கூறப்பட்டுள்ளது. 50 வயதுக்கு மேல் உள்ள பெண்களுக்கு இப்பாதிப்பு அதிகமாய் இருந்தது. ஆனால் இக்காலத்தில் 30 வயதுள்ளோர்க்குக் கூட பாதிப்பு இருக்க கூடும் என மருத்துவர்கள் சுய பரிசோதனை மேற்கொள்ள அறிவுறுத்துகின்றனர். பிறப்பு முதல் இறப்பு வரை பெண் என்பவள் பல பருவங்களை கடக்கிறாள். பொருப்புகளை சுமந்து, பொருமையாக வாழ்ந்து, பிறந்த வீட்டிற்கும், புகுந்த வீட்டிற்கும் பெருமை பல சேர்த்து தன் வாழ்நாளை தனக்கென வாழாமல் பிறர்கெனவே அதிகம் வாழ்வதால் தானே, பெண்ணை தெய்வம் என நம் தமிழ் கலாச்சாரம் கொண்டாடுகிறது. அப்பேற்பட்ட திறன் வாய்ந்த பெண்கள் உடல் உபாதையினால் பாதிக்கப்பட்டால் சீர்குலைவே மிஞ்சும். அதிலும் மார்பகப்புற்று நோய் மிகவும் கொடியது.

\section{மார்பகப் புற்றுநோயின் வரலாறு}

இந்நோய் முதல் முறையாக எகிப்து நாட்டில் கி.மு.1600களில் கண்டுபிடிக்கப்பட்டது. இந்த நூற்றாண்டுகளில் எட்வின் ஸ்மித் பேப்பரஸ் என்பவர் எந்தவித சிகிச்சையும் இந்நோய்க்கு இல்லை என்றும் 8 வகையான கட்டிகள் மற்றும் புண்களைப் பற்றியும் எழுதியுள்ளார். இவரைத் தொடர்ந்து பல நூற்றாண்டுகள் மருத்துவர்கள் இதே முடிவுகளை எழுதினர். மார்பக புற்று நோய்க்கும் அக்குள்களில் உள்ள நிணநீர் முடிச்சுகளுக்குள் உள்ள தொடர்பை 17 ஆம் நூற்றாண்டிற்கு பின்னர் சில மருத்துவர்கள் கண்டறிந்தனர். 1674 ஆம் ஆண்டு பிரஞ்சு அறுவைசிகிச்சை நிபுணர் ஜீன் லூயிஸ் பெடிட் என்பவரும் பின்னர் ஸ்காட்டிஷ் நாட்டை சார்ந்த அறுவைசிகிச்சை நிபுணர் பெஞ்சமின் பெல் என்பவரும் சேர்ந்து முதன்முறையாக மார்பக திசுக்கள், நிணநீர் முடிச்சுகள் மற்றும் அதற்கு கீழுள்ள மார்புதசை ஆகியவற்றை அகற்றினார். 1882 ஆம் ஆண்டுகளில் மாஸ்டெக்டோமி என்ற இரண்டு மார்பகங்களையும், நிணநீர் முடிச்சுகள் மற்றும் கீழே இருக்கும் மார்பு திசைகள் அகற்றம் என்ற நீண்டகாலத்து வலியும், முடங்கியிருக்க கூடிய நிலையுடைய சிகிச்சை நடைமுறையில் இருந்தது. தற்பொழுது உள்ள நவீன மருத்துவமுறைகளில் அறுவைசிகிச்சைக்கு மட்டுமின்றி பல எளிதான சிகிசைசை முறைம் கண்டுபிடிக்கபாட்டுள்ளன.

\section{மார்பகப் புற்றுநோய்க்கான காரணங்களும் காரணிகளும்}

மார்பகப்புற்றுநோய் வருவதற்கான பல காரணங்கள் கூறப்படுகின்றன. அவையாவன;

> பெண்கள் முன்கூட்டியே பருவமடைவதினாலும்

> 30 வயதிற்குப் பிறகு திருமணம் நடப்பதாலும்

> குழந்தைப் பேறின்மைக்கான தொடர் ஹார்மோன் சிகிச்சையினாலும் 
> செயற்கை கருத்தரிப்பு முறையில் (டெஸ்டியூப் பேபி) பிள்ளை பெறுவதாலும்

> ஒழுங்கற்ற மாதவிடாய் போக்கு இருப்பதாலும்

> குரோமோசோம்களில் BRCA 1\&2 மரபணுக்கள் தோன்றுவதாலும்

> மரபணு பிறழ்ச்சி (Genetic Disorder) ஏற்படுவதாலும்

> ஒவ்வாத மேற்கத்திய உணவுப் பழங்களாலும்

> உடற்பயிற்ச்சியின்மை, சோம்பேறித்தனம்

> புகை மற்றும் மது பழக்கம்.

> ஹார்மோன் ஏற்றத்தாழ்வு.

> பிள்ளை பேருக்கு பின்பு பாலுட்டுதல் இல்லாமை என பல்வேறு காரணங்கள்.

\section{மார்பகப் புற்றுநோய்க்கான அறிகுறிகள்:}

மார்பகப் புற்றுநோயானது நான்கு படிநிலைகளைக் கொண்டது. முதல் இரண்டு நிலைகளிலேயே கண்டறியப்பட்டால் இதிலிருந்து மீண்டுவிடலாம்.

> ஆரம்பகாலத்தில் நகரக்கூடியக் கட்டி அல்லது மார்பகத்தில் ஏற்பட்டு பிற்காலத்தில் நிலைப்பெருதல்.

> மார்பகத்தில் சிவந்து காணப்படுதல் மற்றும் வீக்கம்.

> முலைக்காம்பும், மார்பகத்திசுக்களும் உள்ளிழுத்து காணப்படுதல்.

> காம்பில் இரத்தம் அல்லது வெள்ளை நிற திரவக் கசிவு.

> மார்பக புறபரப்பில் புதிதாக புள்ளிகள் தோன்றும்.

> மார்பக அளவு மற்றும் வடிவத்தில் மாற்றம்.

> மார்பகத் தோலில் ஏற்படும் பறுக்கள்.

> மார்பகத்தில் ஏற்படும் வலி, வீக்கம், எரிச்சல், அரிப்பு.

\section{மார்பகப் புற்றுநோய்க்கான பரிசோதனைகள்}

இக்கால பெண்களுக்கு மார்பகப் புற்றுநோய் பற்றிய விழிப்புணர்வு மிகவும் அவசியம். அதிலும் சுய பரிசோதனை முறைகளை கட்டாயம் அறிந்திருக்க வேண்டும்.

> சுயமார்பகப் பரிசோதனை

> மாமோகிராம் பரிசோதனை

> மருத்துவரிடம் மார்பக பரிசோதனை

> காந்த அதிர்வு அலை வரைவு என்னும் எம்.ஆர்.ஐ ஸ்கேன்

> நீர்பரிசோதனை என்ற (FNAC)

> பையாப்சி (Biopsy) என பல நவீன பரிசோதனைகள் உள்ளன.

\section{மார்பகப் புற்றுநோய்க்கான முன் எச்சரிக்கைகள்}

இந்நோய் வருமுன் காக்க வழிவிலக்கு உண்டு, அவையாவன

> வைட்டமின் சி மற்றும் ஏ நிறைந்த உணவை உட்கொள்ளுதல்

> தினசரி உடற்பயிற்சிகள் ஆர்வமாக ஈடுபடுதல் 
> இறுக்கமாக மார்பகத்தை அழுத்தும் உடைகள் அணிவதை தவிர்த்தல்

> மகளிர் நல மருத்துவரிடம் தயக்கமின்றி மார்பக பரிசோதனை செய்துகொள்ளுதல்

> குடும்பத்தில் எவருக்கேனும் மார்பக புற்றுநோய் இருந்தால் கூடுதல் கவனத்தோடு நடந்துக்கொள்ளுதல் போன்ற முன் எச்சரிக்கை நடவடிக்கைகளை கடைபிடித்தல் மிகவும் இன்றியமையாத ஒன்றாகும்.

மார்பகப் புற்றுநோய் தரவரிசை

மார்பகப் புற்றுநோயின் உலக தரவரிசையை பார்க்கையில் லட்சத்தில் 113.2 பேர் பாதிக்கப்பட்டு பெல்ஜியம் நாடு முதல் இடத்திலும் லக்ஸம்பர்க் (109.3), நெதர்லாந்து (105.9), பிரான்ஸ் பெருநகர் (99.1), புதிய கலிபோனியா (பிரான்ஸ்) (98.0) ஆகிய நாடுகள் பாதிப்பு விகிதத்தில் முதல் ஐந்து இடங்களில் உள்ளன (Meesha Chaturvedi et al., 2015; Bharath Rangarajan et al., 2016; Carol E. DeSantis et al., 2016; ).

அட்டவணை 1. உலகப் புற்றுநோய் புள்ளிவிவரம்

\begin{tabular}{|l|l|l|}
\hline கண்டங்கள் & $\%$ & மொத்த எண்ணிக்கை \\
\hline அமெரிக்கா & $21.0 \%$ & 3792000 \\
\hline ஆபிரிக்கா & $5.8 \%$ & 1055000 \\
\hline ஐரோப்பா & $23.4 \%$ & 4230000 \\
\hline ஆசியா & $48.4 \%$ & 8751000 \\
\hline ஓசனியா & $1.4 \%$ & 252000 \\
\hline & & $\begin{array}{l}\text { பாதிப்பு -18.1 மில்லிய } \\
\text { இறப்பு - } 9.6 \text { เில்லிய }\end{array}$ \\
\hline
\end{tabular}

Source: (WHO,2018 Global cancer data: Globocan, Geneva, Switzerland)

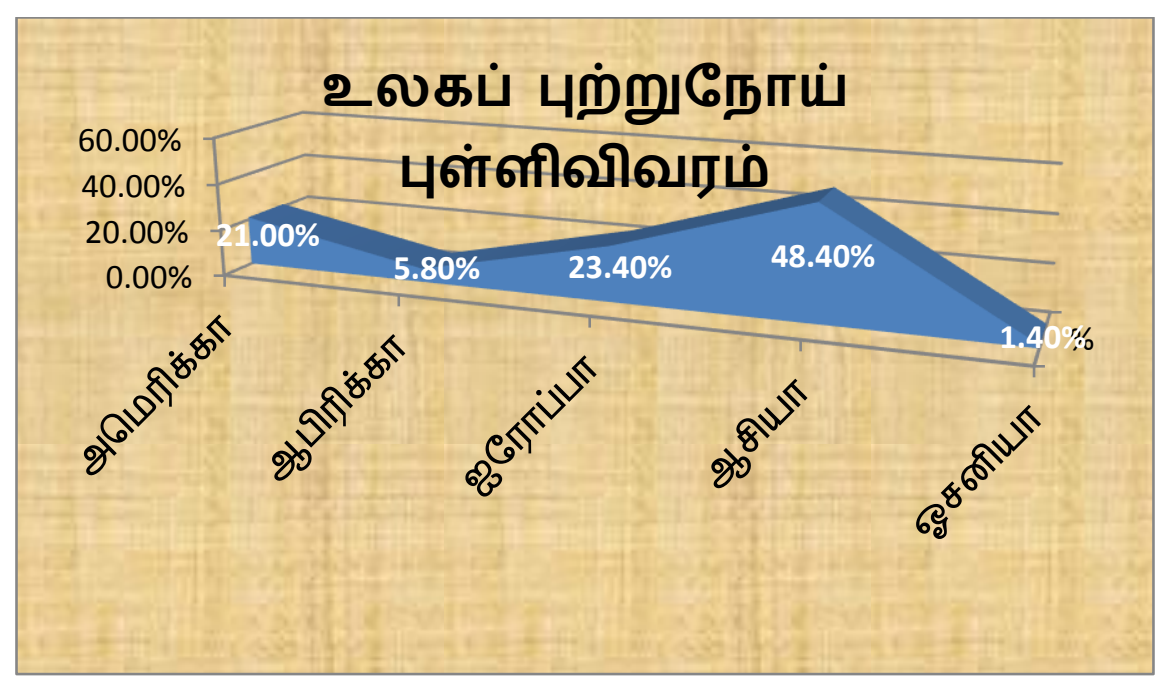

படம் 1. உலகப் புற்றுநோய் புள்ளிவிவரம் 


\section{இந்தியாவில் மார்பகப்புற்றுநோய்}

மற்றப் புற்றுநோய்களைக் காட்டிலும் இந்திய பெண்களின் மத்தியில் மார்பகப் புற்றுநோய் முதலிடத்தில் உள்ளது. (14\%) பாதிப்பு விகிதம் லட்சத்தில் 25.8 ஆகவும், இறப்பு விகிதம் லட்சத்தில் 12.7\% ஆகவும் உள்ளது. இந்தியாவில் ஒவ்வொரு 4 நிமிடங்களுக்கும் ஒரு பெண் மார்பகப் புற்றுநோயால் பாதிக்கப்படுகிறார். மேலும் ஒவ்வொரு 13 நிமிடங்களுக்கும் ஒரு பெண் மார்பகப் புற்றுநோயால் இறந்தும் விடுகிறார் (Javaid sofi, 2020). சரியான வயதில் திருமணம், குழந்தை பிறப்பு, குழந்தைகளுக்கு தாய்ப்பால் ஊட்டுதல், கடுமையான உடல் வேலை ஆகியவை மார்பகப் புற்றுநோய் வராமல் தடுப்பதற்கான காரணிகள் ஆகும். 2018 ஆம் ஆண்டின் கணக்கெடுப்பின் படி 1,62,468 பெண்கள் புதிதாக மார்பகப் புற்றுநோயினால் பாதிக்கப்பட்டு அதில் $\quad 87,090$ பேர் உயிரிழந்தனர். தென்னிந்தியாவில் 531 பெண்களுக்கு மேற்கொண்ட ஆய்வின் முடிவில் கிராமப் புற பெண்களைவிட நகர்ப் புற பெண்களே மார்பகப் புற்றுநோயால் அதிகம் பாதிக்கப் படுகின்றனர் எனக் கண்டறியப்பட்டுள்ளது. அது மட்டுமின்றி 50 வயதிற்கு மமல் மாதவிடாய் நிற்பது, 13 வயதிற்கு முன்பு பூப்படைதல், புற்றுநோய் ஏறற்படுத்தக்கூடிய மரபணுக்கள், மற்றும் 25 வயதிற்கு மேல் முதல் கர்ப்பம் ஆகியவை முக்கிய காரணிகளாக கண்டறிப்பட்டன.

அட்டவணை 2. இந்தியப் புற்றுநோய் புள்ளிவிவரம் (Javaid Sofi, 2020)

\begin{tabular}{|l|l|l|}
\hline புற்று நோய்கள் & $\%$ & மொத்த எண்ணிக்கை \\
\hline மார்பகப் புற்றுநோய் & $26.3 \%$ & 178361 \\
\hline கருப்பை வாய் புற்றுநோய் & $18.3 \%$ & 123907 \\
\hline கருப்பை புற்றுநோய் & $6.7 \%$ & 45701 \\
\hline உதடு, வாய் புற்றுநோய் & $4.6 \%$ & 31268 \\
\hline பெருங்குடல் புற்றுநோய் & $3.7 \%$ & 24950 \\
\hline இதரப் புற்றுநோய்கள் & $40.4 \%$ & 274196 \\
\hline & & 678383 \\
\hline
\end{tabular}

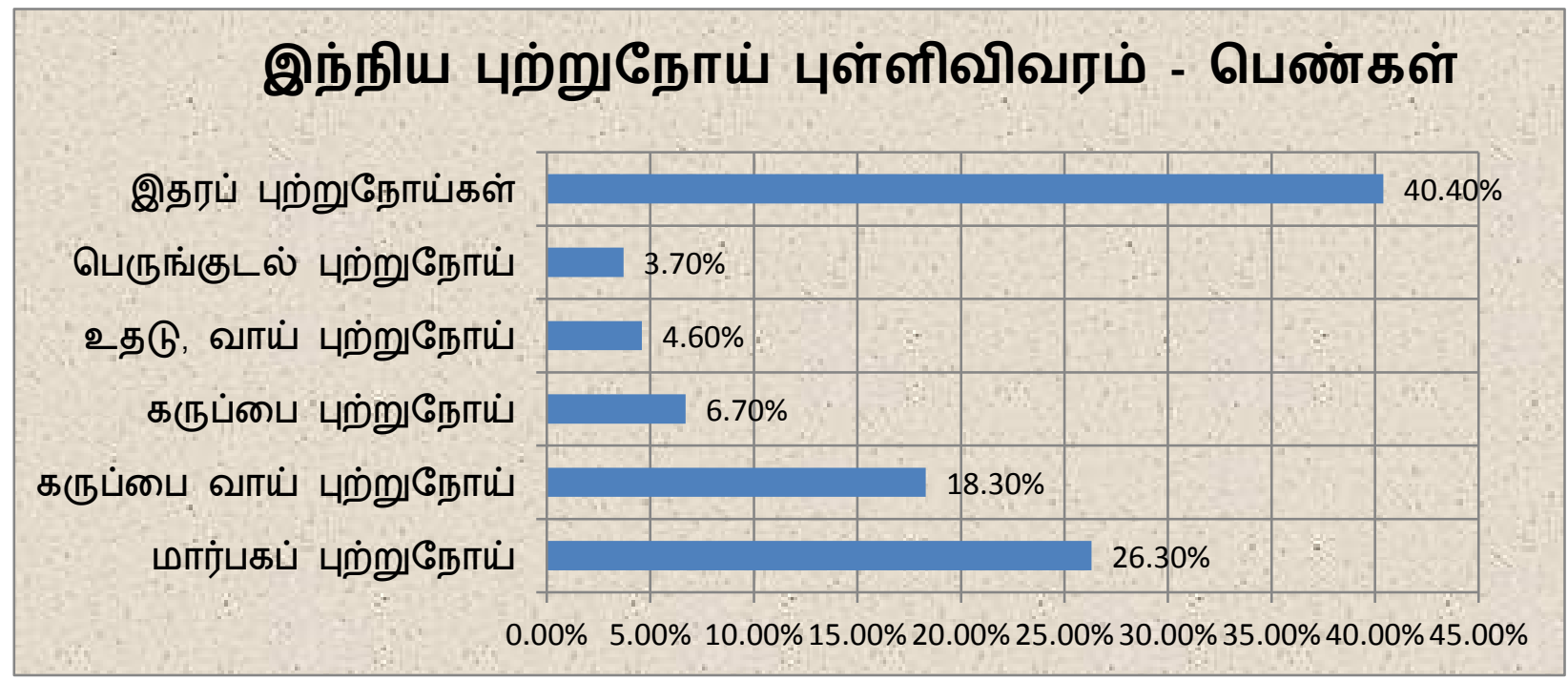

படம் 2. இந்நிய புற்றுநோய் புள்ளிவிவரம் - பெண்கள் 


\section{மார்துளைத்த புற்றுநோயினால் மனமுடைந்த பெண்கள்}

மார்பகம் உடலின் ஒரு பாகம் தானே என சுலபமாய் எண்ணமுடியாது. சிறுநீரகமோ, கண்ணோ ஒன்றை இழந்தாலும் மற்றொன்றை வைத்து சமாளித்து வாழ இயலும். ஆனால் பெண்ணின் வாழ்வில் மார்பகங்களுக்கு பெரும் இடம் உள்ளது அழகு சார்ந்த விஷயம் என்பது மட்டுமின்றி பிள்ளைப்பேறு, தாம்பத்தியம் என பல காரணிகள் இருப்பதாலும் இந்நோயுற்றோர் மகவவும் மனமுடைந்து போகின்றனர். ததனால் பல குடும்பங்களில் நிம்மதியற்ற நிலையும் காணப்படுகிறது. இந்நோய் தாக்கத்தினால் வரும் வலியைவிட, கீமோதெரபியின் வலியை விட மன உளைச்சலும், மன வலியுமே அதிகம். இலக்கியங்களில் பெண்ணை வர்ணிக்கையில் கூந்தலும், முக அழகும், கொடியிடை, நளினம், குணம், பொருமை, மார் அழகும் இல்லா கவியுன்டோ? சங்க இலக்கியத்தில் 168 இடங்களில் முலை என்னும் பெயர் கொண்டும் இரண்டு இடங்களில் கொங்கை என்னும் பெயர் கொண்டும் பரிபாடல், திருமுகாற்றுப்படை, பொருநராற்றுப்படை, சிறுபணாற்றுப்படை, பெரும்பணாற்றுப்படை, மதுரைக்காஞ்சி, நெடுநல்வாடை, நற்றிணை, குறுந்தொகை, ஐங்குறுநூறு, பதிற்றுப்பத்து, புறநானூறு, அகநானூறு என அனைத்து இலக்கியங்களிலும் மார்பகங்கள் வர்ணிக்கப்பட்டுள்ளது. இத்தகு பெருமைக்குரிய பெண்ணின் அடையாளத்திற்கே பாதிப்பு ஏற்படுவதால் மார்பகப்புற்றுநோய் வெறும் உடல் சார்ந்த நோயாக மட்டுமின்றி மனம்சார்ந்த பெரும் பாதிப்பை ஏற்படுத்தப்புகிறது.

\section{மார்பக புற்றுநோயால் பாதிகபட்டவர்களின் வாழ்வில் ேோகாவின் பங்களிப்பு}

யோகாவிற்கு பிறகு மார்பு புற்றுநோயால் பாதிக்கப்பட்டோர் தங்களின் செயல்பாடுகளில் தடைகள் குறைக்கப்பட்டன மற்றும் உடற்பயிற்சி மேம்படுத்தப்பட்டதும் கண்டறியப்பட்டுள்ளது மற்ற துணை சிகிச்சை முறைகளுடன் ஒப்பிடும் போது கீமோதெரபி தூண்டப்பட்ட பக்க விளைவுகளை குறைப்பதில் யோகா நன்மை பயக்கும் என்று நிரூபிக்கப்பட்டுள்ளது. ேோகா நிகழ்ச்சிகள் பங்கேற்ற பிறகு மார்பகப் புற்றுநோயால் பாதிக்கப்பட்ட பெண்கள் தங்கள் மனநிலையில் முன்னேற்றத்தை கண்டுள்ளனர்.

மார்பகப் புற்றுநோய் சிகிச்சைக்கு உட்படுத்தும்போது அவர்கள் குறைந்த சோர்வு நிலையை உணர்ந்துள்ளனர். மார்பகப் புற்றுநோயாளிகளுக்கான யோகா திட்டங்கின் ஆய்வுகள் உணர்ச்சி விளைவுகளிலும் வாழ்க்கைத் தரத்திலும் முன்னேற்றம் கண்டுள்ளனர். சிகிச்சையினால் ஏற்படும் எதிர்மறை மற்றும் நேர்மறை பாதிப்புகள் குறைவதையும் துணை சிகிச்சையினால் ஏற்படும் கவலை மற்றும் மனச்சோர்வு குறைவதையும் ஆராய்ச்சியின் மூலம் பல ஆசிரியர்கள் விவரித்துள்ளனர். (F. Siedentopf et al., 2013) மார்பகப் புற்றுநோய் சிகிச்சையின் போது உளவியல் ஆரோக்கியத்தை மேம்படுத்துவதற்காக போக பயிற்சிகளை மருத்துவர்கள் பரிந்துரைக்கலாம் என தனது ஆராய்ச்சியின் மமலம் (Julienne E. Bower et al., 2014) ஆதாரப்பூர்வமாக தெரிவிக்கிறார். லென்காச்சரும் (2016) அவரது சாகாக்களும் சேர்ந்து மார்பகப் புற்றுநோயால் பாதிக்கப்பட்டு சிகிச்சை பெற்றவர்களுக்கு முதலில் ஆறு வாரம் மற்றும் பிறகு 12 வாரங்களுக்கு தியான பயிற்சிகளை நான்கு நுட்பங்களாக வழங்கினர். அவையாவன 
சுவாசத்தை கவனித்தல் தன் முழு உடலையும் அறிந்து கொள்ளுதல் யோக பயிற்சி மற்றும் நடைப்பயிற்சி தியானம் ஆகியவை அடங்கும். இப்பயிற்சிக்கு 322 பெண்கள் தேர்ந்தெடுக்கப் பட்டு அதில் 299 பெண்கள் வெற்றிகரமாக முடித்தனர். இதன் விளைவு சிறியது முதல் பெரியது வரை கண்டுபிடிக்கப்பட்டது. அவையாவன, மீண்டும் மீண்டும் ஏற்படும் அச்சம், சோர்வு, மன உளைச்சல் ஆகியவை குறைந்த காணப்பட்டன. அவர்கள் தங்கள் தொடர்பயிற்சி காரணமாக உணர்ச்சிகளை தாங்களே கட்டுப் படுத்த கற்றுக்கொண்டுள்ளனர். சுருக்கமாக நினைவாற்றல் அடிப்படையிலான தலையீடு இளைய மார்பக புற்றுநோயால் தப்பிப் பிழைப்பவர்களில் மன அழுத்தம், நடத்தை அறிகுறிகள் மற்றும் அழற்சிக்கு சார்பான சமிக்ஞை ஆகியவற்றை குறைப்பதில் பூர்வாங்க குறுகியகால செயல்திறனை காட்டியுள்ளது. மாதவிடாய் நின்ற மார்பக புற்றுநோயால் கண்டறியப்பட்ட பெண்களுக்கு நீண்ட காலம் உயிர் வாழக்கூடிய காலப்பகுதியில் உயர்ந்த அளவு மன அழுத்தம் மன உளைச்சல் மற்றும் உடல் அறிகுறிகளை நிர்வகிக்க உதவும் உத்திகள் தேவைப்படுகின்றன. அவ்வாறான உத்திகளில் முக்கிய பங்கு வகிப்பது தியான பயிற்சியாகும். இதன் தலையீடு இந்த பெண்களுக்கு சில குறுகியகால நன்மைகளை வழங்கக்கூடும். அவையாவன உளவியல், நடத்தை மற்றும் உயிரியல் விளைவுகளில் மேம்பாடு என்று இந்த ஆராய்ச்சியின் மூலம் கண்டுபிடிக்கப்பட்டுள்ளது. இப்பயிற்சிகளை அப்பெண்கள் தன் வாழ்நாளில் தொடர்ந்தால் சாத்தியமான நன்மைகள் ஏற்படும் என கூறுகிறார்.

\section{முடிவுரை}

நாம் சேமித்து வைக்கும் நகை, பணம், வீடு, நிலம் முதலியன உண்மையான சொத்துக்கள் அல்ல. நோயற்ற வாழ்வே குறைவற்ற செல்வம் கலாம் ஐயா கூறியது போல் பெண்ணே ஒவ்வொரு குடும்பத்தின் ஆணிவேர். மார்பகப் புற்றுநோயை முன்கூட்டியே கண்டறிந்தால் உறுதியாக மீண்டுவிடலாம். கவனக்குறைவாலும், அலட்சியத்தாலும் அறியாமையாலுமே இ இந்நோய் பெரிதாகி உள்ளது. பெண்களின் மத்தியில் போதிய விழிப்புணர்வு இருந்தாலே இந்நோயை விரட்டி அடித்துவிடலாம்.

\section{References}

Carol E. DeSantis, Stacey A. Fedewa, Ann Goding Sauer, Joan L. Kramer, Robert A. Smith, Ahmedin Jemal, (2016), Breast cancer statistics, 2015: Convergence of incidence rates between black and white women, CA Cancer Journal for Clinicians, 66(1) 31-42.

Javaid Sofi (2020) Breast Cancer in India 12:28 PM IST in Poverty of Ambition, India, TOI https://timesofindia.indiatimes.com/blogs/poverty-of-ambition/breast-cancer-in-india/ (Accessed on June 2020)

Julienne E. Bower, Alexandra D. Crosswell, Annette L. Stanton, Catherine M. Crespi, Diana Winston, Jesusa Arevalo, Jeffrey Ma, Steve W. Cole, Patricia A. Ganz, (2015) Mindfulness meditation for younger breast cancer survivors: A randomized controlled trial, Cancer, 121(8) 1231-1240. https://doi.org/10.1002/cncr.29194

Meesha Chaturvedi, Vaitheeswaran, K., Satishkumar, K., Priyanka Das, Stephen, S., and Nandakumar, A., (2015) Time Trends in Breast Cancer Among Indian Women Population: An Analysis of Population 
Based Cancer Registry Data, Indian Journal of Surgical Oncology, 6(4) 427-434. https://dx.doi.org/10.1007/s13193-015-0467-z

Siedentopf, F., Utz-Billing, I., Gairing, S., Schoenegg, W., Kentenich, H., Kollak I., (2013) Yoga for Patients with Early Breast Cancer and its Impact on Quality of Life - a Randomized Controlled Trial, Geburtshilfe Frauenheilkd, 73(4) 311-317. https://dx.doi.org/10.1055/s-0032-1328438

Tanuja Shet, Tabassum Wadasadawala, Nita S. Nair, R. Madhu Sairam, Sachin S. Hingmire and Jyoti Bajpai, (2016) Breast cancer: An overview of published Indian data, South Asian Journal of Cancer, 5(3) 8692. https://dx.doi.org/10.4103/2278-330X.187561

WHO, (2018) Global cancer data: Globocan, Geneva, Switzerland https://www.uicc.org/news/globalcancer-data-globocan-2018 (Accessed on April 2020)

\section{Funding: NIL}

Acknowledgement: NIL

Conflict of Interest: NIL

\section{About the License:}

\section{(c) (1)}

Attribution 4.0 International (CC BY 4.0)
(C) The authors 2021. The text of this article is licensed under a Creative Commons Attribution 4.0 International License 